\title{
Biological Properties of Cells Other Than HSCs
}

\author{
Attilio Bondanza, Ulrike Koehl, Andrea Hoffmann, \\ and Antoine Toubert
}

\subsection{Introduction}

The array of cellular players involved in the biology of HSCT clearly extends beyond HSC themselves and, in the case of transplantation from allogeneic sources, importantly includes cells of the innate and adaptive immune system. Historically, the discovery of the HLA system and the functional characterization of the different immune cell types had a transformational impact on our current understanding of the pathobiological sequelae of allo-HSCT (rejection, GVHD, the GVL effect). This body of knowledge coupled to the most recent exploit of biotechnology nowadays allows us to design strategies for in vivo stimula-

A. Bondanza $(\bowtie)$

Innovative Immunotherapies Unit, Division of

Immunology, Transplantation and Infectious

Diseases, University Vita-Salute San Raffaele and

Ospedale San Raffaele Scientific Institute, Milan, Italy

e-mail: Bondanza.Attilio@hsr.it

U. Koehl

University Hospital and Fraunhofer IZI, Leipzig,

Germany

Hannover Medical School, Hannover, Germany

A. Hoffmann

Department of Orthopaedic Surgery, Laboratory of Biomechanics and Biomaterials, Hannover Medical School, Hannover, Germany

A. Toubert

University Paris Diderot and Hopital Saint Louis,

Paris, France tion or adoptive transfer of specific immune cell types with the potential to dramatically improve transplantation outcome.

In this chapter, we will review the biological properties of cells other than HSCs that so far have Since apart from vaccination antigen presenting cells and myeloid cells at large have seldom been subject of this type of studies been therapeutically investigated in human allo-HSCT, they will not be discussed here. Conversely, we will briefly touch on mesenchymal stromal cells (MSCs), which, although not classifiable as immune cells stricto sensu, have been widely employed in allo-HSCT.

\subsection{Conventional or Alpha-Beta T Cells}

The majority of mature $\mathrm{T}$ cells is characterized by the expression of the $\alpha \beta$ TCR, which endows MHC-restricted recognition of peptides derived from non-self-proteins. Mutually exclusive coexpression of CD8 or CD4 further conveys specificity for MHC class I/MHC class II/peptide complexes, respectively. CD8+ T cells recognize intracellular peptides, mainly derived from viruses or mutated genes, mediating cytotoxicity of infected or transformed cells, thence the name cytotoxic T lymphocytes (CTLs). Conversely, CD4+ T cells recognize extracellular pathogenderived peptides, providing antigen-specific 
specific "help" to bystander immune cells, such as B cells in antibody production and phagocytes in killing of engulfed pathogens. Alloreactivity occurs because of $\alpha \beta$ TCR-mediated recognition of mismatched HLAs or of non-HLA polymorphic peptides presented in the context of matched HLAs, e.g., those derived from H-Y (malespecific histocompatibility antigen). The latter are known as minor histocompatibility antigens (mHag) and play a major role in GVHD and the GVL effect after HLA-matched transplantation.

The adoptive transfer of CTLs specific for important opportunistic viruses in allo-HSCT (CMV, EBV, ADV) has been one of the first manipulated cellular immunotherapies to be tested in humans (Bollard and Heslop 2016) and in some EU countries is now available as an offthe-shelf therapy from HLA-matched donors. Conversely, it has been proposed that naïve $\mathrm{T}$ cells, i.e., cells that have never encountered their cognate antigen, may be more alloreactive than memory $\mathrm{T}$ cells, i.e., antigen-experienced cells that have persisted after clearing the infection. This concept is at the basis of protocols for the depletion of naïve $\mathrm{T}$ cells from the graft as a way to prevent GVHD while retaining a strong GVL effect (Bleakley et al. 2015). Promising are also attempts at translating this approach against hematological tumor antigens for treating overt leukemia relapse after allo-HSCT (Chapuis et al. 2013). On a different page, given the overall complexity of immune responses, it is not surprising that during evolution, some immune cell types have evolved with the specific task of immune regulation. T regulatory cells (Tregs) are thymusderived cells characterized by constitutive expression of the transcription factor FoxP3. Tregs are potent suppressors of alloreactivity and are now being investigated for GVHD management after their ex vivo expansion (Brunstein et al. 2016).

\subsection{Unconventional T Cells}

Unconventional $\mathrm{T}$ cells include $\mathrm{T}$ cells expressing the $\gamma \delta$ TCR, invariant natural killer T cells (iNKT) cells, and mucosal-associated invariant (MAIT) $\mathrm{T}$ cells-which will not be treated here-and are an abundant component of the immune system. Although originating from the thymus, they all share lack of MHC-restricted peptide recognition and mainly reside within epithelial tissues. They have a limited TCR repertoire diversity and get activated quickly, bridging innate to adaptive immunity.

1. A subset of $\gamma \delta \mathrm{T}$ cells $(\mathrm{V} \gamma 2 \mathrm{~V} \delta 9)$ are activated by phosphoantigens, non-peptidic metabolites produced by mammalian cells and intracellular pathogens (M. tuberculosis, M. leprae, Listeria species, Plasmodium species) after interacting with intracellular butyrophilin 3A1. Gammadelta $\mathrm{T}$ cells can also recognize stress molecules such as MICA, MICB, and ULBPs through the NK receptor NKG2D. The possibility to expand $\mathrm{V} \gamma 2 \mathrm{~V} \delta 9$ effector T cells in vivo by administering the therapeutic bisphosphonate zoledronate has originated many clinical trials in hematological tumors, also in the context of transplantation (Airoldi et al. 2015).

2. Type I invariant NKT is a distinct population of $\alpha \beta$ T cells characterized in humans by the expression of $\alpha 24-\mathrm{J} \alpha 18$ preferentially paired to $\mathrm{V} \beta 11$. They recognize lipids presented in the context of broadly distributed CD1d (monocytes/macrophages, B cells, epithelial cells). Upon activation, iNKT cells produce immune regulatory cytokines and kill tumor targets. Failure to reconstitute iNKT cells after Allo-HSCT (Rubio et al. 2012) or lower iNKT cells in the graft (Chaidos et al. 2012) has been linked to GVHD and relapse. Alphagalactosyl ceramide is a marine spongederived lipid antigen known to expand iNKT cells in vivo and is currently under investigation in Allo-HSCT (Chen et al. 2017).

\subsection{NK Cells}

Natural killer (NK) cells belong to the innate immune system and provide immediate reactivity against virally infected, as well as tumor targets. NK cytotoxicity is controlled by a balance of several germ-line encoded inhibitory and activating receptors, such as killer immunoglob- 
ulin-like receptors (KIRs) and natural cytotoxicity receptors (Vivier et al. 2011). The importance of NK cells in allo-HSCT has surfaced after the demonstration of their pivotal role in preventing leukemia relapse and decreasing GVHD risk after grafting from HLAhaploidentical donors (Ruggeri et al. 2002). Since then, there has been a growing interest in using both autologous and allogeneic NK cells in patients with leukemia or other high-risk hematological tumors, also in the non-transplant setting (Koehl et al. 2016). These trials have uniformly shown safety and potential efficacy of infused NK cells. Nevertheless, they have also documented the emergence of powerful immune escape mechanisms, raising the question on how to improve NK cell-based therapies (Koehl et al. 2018). Various trials are under way in order to investigate ways to achieve better NK cell cytotoxicity and overcome the immunosuppressive tumor microenvironment, including:

1. Combination of novel checkpoint inhibitors with activated NK cells

2. Bi- or tri-specific antibodies for directly binding NK cells to cancer cells

3. Chimeric antigen receptor (CAR)-modified NK cells for direct targeting of cancer cells

The latter strategy is particularly interesting since CAR-NK cells are expected to retain their natural antitumor reactivity, opening for potentially synergistic effects. The first clinical CAR-NK cell studies targeting CD19 and NKG2D ligands have been initiated (ClinGov. No NCT03056339, NCT01974479, NCT00995137, NCT03415100) and will likely be instrumental to demonstrate proof of concept.

\subsection{Mesenchymal Stromal Cells}

Mesenchymal stroma cells (MSCs) are multipotent cells capable of differentiating into cells and tissues of the mesodermal lineage (bone, cartilage, and adipose cells) (Pittenger et al. 1999). Apart from their regenerative properties, MSCs have been discovered to secrete a variety of soluble factors and exosomes with paracrine actions. Instead of focusing on MSC regenerative properties, most clinical studies have investigated their immunomodulatory (often immunosuppressive) properties, as well as their trophic influence on tissue repair, especially in GVHD (Fibbe et al. 2013). Interestingly, subsequent to hematopoietic stem cells, MSCs are the second most frequently used cell source for therapeutic applications. Notwithstanding their widespread use, MSCs are currently the stem cell population with the least defined identity and properties (Hoffmann et al. 2017).

Important studies have demonstrated that the physiological counterpart of ex vivo-expanded MSCs can be both CD146+ adventitial reticular cells in the subendothelial layer of microvessels (Tormin et al. 2011) and CD146- pericytes surrounding large vessels (Corselli et al. 2013). MSC biological functions are also highly debated and conflicting results were reported in vitro and, more importantly, in clinical trials (Fibbe et al. 2013). Considerable lack of consensus exists within the field as to how MSCs exert their multipronged effects. This is due to several facts: Firstly, MSCs are isolated from many tissues and by different protocols. Secondly, due to the mode of isolation, these cells present heterogeneous cell populations. Thirdly, protocols for in vitro expansion, including the culture conditions (culture vessels, media, additives, passaging), are different. Fourthly, MSCs have often been reported to survive in vivo only for short time (days). A recent comparison of MSC preparations from eight different centers using BM aspirates as starting material for GMP-guided processes revealed considerable variability between the centers (Liu et al. 2017). Cells from six centers were compared in vivo for bone formation and hematopoiesis support. The quantity of deriving bone was highly variable, and only MSCs from three centers supported hematopoiesis. A critical reappraisal of these cell populations and harmonization of the methods for their isolation and expansion, as well as the development of validated potency assays, is therefore necessary for harnessing their full therapeutic potential. 


\section{Key Points}

- HSCT rather than a solo play is an orchestral concert, where different cellular players contribute to the overall final result of the symphony.

- Besides obviously HSCs, key contributors are cells of the innate and adaptive immune system. Both have evolved for the key task of self/non-self-discrimination, each however focusing on the recognition of different class of molecules, from proteins to glycolipids.

- The tremendous knowledge in immunobiology acquired in the last few decades has enabled to start exploiting the properties of these cells or ameliorating the outcome of HSCT.

\section{References}

Airoldi I, Bertaina A, Prigione I, et al. $\gamma \delta$ T-cell reconstitution after HLA-haploidentical hematopoietic transplantation depleted of TCR- $\alpha \beta+/ C D 19+$ lymphocytes. Blood. 2015;125:2349-58.

Bleakley M, Heimfeld S, Loeb KR, et al. Outcomes of acute leukemia patients transplanted with naive T cell-depleted stem cell grafts. J Clin Invest. 2015;125:2677-89.

Bollard CM, Heslop HE. T cells for viral infections after allogeneic hematopoietic stem cell transplant. Blood. 2016;127:3331-40.

Brunstein CG, Miller JS, McKenna DH, et al. Umbilical cord blood-derived $\mathrm{T}$ regulatory cells to prevent GVHD: kinetics, toxicity profile, and clinical effect. Blood. 2016;127:1044-51.

Chaidos A, Patterson S, Szydlo R, et al. Graft invariant natural killer T-cell dose predicts risk of acute graftversus-host disease in allogeneic hematopoietic stem cell transplantation. Blood. 2012;119:5030-6.

Chapuis AG, Ragnarsson GB, Nguyen HN, et al. Transferred WT1-reactive CD8+ T cells can mediate antileukemic activity and persist in post-transplant patients. Sci Transl Med. 2013;5:174ra27.
Chen Y-B, Efebera YA, Johnston L, et al. Increased Foxp3+Helios+ regulatory $\mathrm{T}$ cells and decreased acute graft-versus-host disease after allogeneic bone marrowtransplantation in patients receiving sirolimus and RGI-2001, an activator of invariant natural killer T cells. Biol Blood Marrow Transplant. 2017;23:625-34.

Corselli M, Crisan M, Murray IR, et al. Identification of perivascular mesenchymal stromal/stem cells by flow cytometry. Cytom Part J Int Soc Anal Cytol. 2013;83:714-20.

Fibbe WE, Dazzi F, LeBlanc K. MSCs: science and trials. Nat Med. 2013;19:812-3.

Hoffmann A, Floerkemeier T, Melzer C, Hass $\mathrm{R}$. Comparison of in vitro-cultivation of human mesenchymal stroma/stem cells derived from bone marrow and umbilical cord. J Tissue Eng Regen Med. 2017;11:2565-81.

Koehl U, Kalberer C, Spanholtz J, et al. Advances in clinical NK cell studies: donor selection, manufacturing and quality control. Oncoimmunology. 2016;5:e1115178.

Koehl U, Toubert A, Pittari G. Editorial: tailoring NK cell receptor-ligand interactions: an art in evolution. Front Immunol. 2018;9:351.

Liu S, de Castro LF, Jin P, et al. Manufacturing differences affect human bone marrow stromal cell characteristics and function: comparison of production methods and products from multiple centers. Sci Rep. 2017;7:46731.

Pittenger MF, Mackay AM, Beck SC, et al. Multilineage potential of adult human mesenchymal stem cells. Science. 1999;284:143-7.

Rubio M-T, Moreira-Teixeira L, Bachy E, et al. Early posttransplantation donor-derived invariant natural killer T-cell recovery predicts the occurrence of acute graft-versus-host disease and overall survival. Blood. 2012; 120:2144-54.

Ruggeri L, Capanni M, Urbani E, et al. Effectiveness of donor natural killer cell alloreactivity in mismatched hematopoietic transplants. Science. 2002;295:2097-100.

Tormin A, Li O, Brune JC, et al. CD146 expression on primary nonhematopoietic bone marrow stem cells is correlated with in situ localization. Blood. 2011;117:5067-77.

Vivier E, Raulet DH, Moretta A, et al. Innate or adaptive immunity? The example of natural killer cells. Science. 2011;331:44-9.

Open Access This chapter is licensed under the terms of the Creative Commons Attribution 4.0 International License (http://creativecommons.org/licenses/by/4.0/), which permits use, sharing, adaptation, distribution and reproduction in any medium or format, as long as you give appropriate credit to the original author(s) and the source, provide a link to the Creative Commons license and indicate if changes were made.

The images or other third party material in this chapter are included in the chapter's Creative Commons license, unless indicated otherwise in a credit line to the material. If material is not included in the chapter's Creative Commons license and your intended use is not permitted by statutory regulation or exceeds the permitted use, you will need to obtain permission directly from the copyright holder. 